\title{
LES PRODUCTIONS SIMULTANÉES DE MIEL, GELÉE ROYALE ET ABEILLES, SONT-ELLES INCOMPATIBLES?
}

PAR

\author{
M. VUILLA UME
}

Station de Recherches Apicoles, Bures-sur-Yvette

Depuis I950, certains apiculteurs producteurs de miel ont plus ou moins délaissé cette production en vue de celle de gelée royale.

I es techniques alors utilisées (starter et finisseur, ou orphelinage de la ruche) perturbaient, il est vrai, considérablement les ruches, et les abeilles récoltaient juste assez de provisions pour les colonies.

Les apiculteurs croyaient, grâce à un nourrissement intensif, augmenter leur production de gelée royale. Cet apport quotidien de sucre les empêchait de placer des hausses dans lesquelles les Abeilles auraient emmagasiné un mélange de sucre et de miel. D’autre part, si ce nourrissement était nécessaire dans certains cas (début de la production de gelée royale, alors que les colonies n'occupent pas tout le volume de la ruche, ou bien pendant des périodes où le nectar manque à l'extérieur) dans d'autres cas il est néfaste. S'ajoutant aux apports de nectar, il contribue à provoquer un blocage de ponte. Or la gelée royale est secrétée par les jeunes Abeilles. Leur nombre dépend directenent de l'intensité de la ponte de la reine, limitée elle-même par la place laissée libre sur les cadres par les Abeilles. Un tel blocage de ponte peut se produire naturellement au cours de fortes miellées et tous les apiculteurs producteurs de gelée royale ont observé les effets néfastes des miellées sur cette production.

Depuis I955, notre programme de recherches portant sur la psychophysiologie de l'élevage des reines chez les Abeilles, nous avons été amenés à utiliser diverses techniques apicoles, en particulier les techniques d'élevage de reines et de production de gelée royale.

\section{Diverses étapes de nos techniques.}

Au cours d'une première étape, les cupules garnies de larves étaient placées dans un "starter o ou paquet d'abeilles sans reines. Elles y séjournaient 24 heures après quoi nous les transposions dans la partie orphe- 
line d'un "finisseur ". Par la suite, nous avons placé directement les larves à élever dans le finisseur (partie orpheline). Rappelons que le finisseur se compose d'une très forte ruche divisée en deux parties :

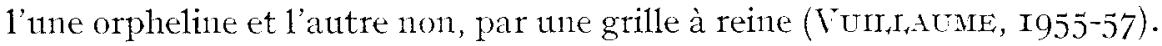

Les colonies ainsi dirigées manifestent une forte tendance à l'essaimage que nous essayions d'empêcher ou de limiter en augmentant le volume de la ruche. Des ruches Dadant I7 cadres ont été construites à cet effet et utilisées au début de nos recherches comme finisseurs. Ce sont ces ruches qui par la suite ont servi à l'élevage complet des cellules royales. Itvitant les blocages naturels de ponte au cours des fortes miellées, elles sont évidemment très aptes à la production de gelée royale. Mais ne peut-on procéder autrement?

\section{Le nourrissement et la production de gelée rọale.}

Nous nous sommes rendu compte que pendant les fortes miellées le nourrissement était superflu, voire même gênant. Après l'avoir supprimé, nous avons pu retirer de nos grandes ruches, des cadres entiers de miel

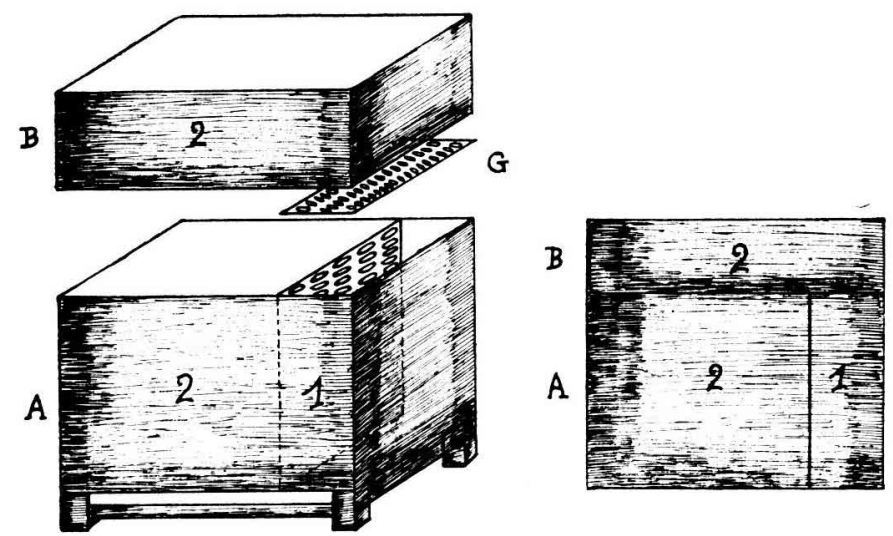

Frg. I. - l'erspetive et profil d'une ruche Dadant i 2 cadres gamie d'une hausse. 2 grilles à reines isolent une partie orphlieline (I) d'une partie non orpheline $(2) .(4=\operatorname{corps}$ de ruche; $R=$ hausse ; $G=$ grille à reine).

operculé. Nous avons alors adapté notre technique à des ruches Iadant Io cadres et I2 cadres, en laissant à la reine libre accès dans la hausse. Deux grilles à reine, l'une horizontale, l'autre verticale (fig. I), lui interdisaient l'accès à la partie orpheline dans laquelle étaient élevées les cellules royales. I a grille verticale, comme dans le finisseur initial, divise le corps de la ruche en 2 parties, l'une avec reine, l'autre sans reine (voir fig. I). Cette dernière partie est isolée de la hausse (où la reine a libre accès) par une grille à reine horizontale. L'espace que nous donnions au- 
paravant en latéralité, la colonie le tronve ici en hauteur. De cette hausse, l'apiculteur peut retirer, puisqu'ils ne contiennent plus de sucre de nourrissement, les cadres de miel. Un inconvénient demeure : il faut à chaque introduction et retrait des cellules royales, écarter la hausse. Cet inconvénient est minime car il permet aux apiculteurs d'utiliser pour la production de gelée royale, un matériel qu'au début, on croyait inadapté.

On peut d'ailleurs le suppriner si, sur des ruches Dadant i 2 cadres, on place des hausses moins larges, ne couvrant que la partie non orpheline de la ruche (fig. 2). La nécessité d'avoir dans la partie orpheline du couvain non operculé, oblige l'apiculteur à effectuer 111 transfert fréquent de cadres d'une partie dans 1'autre. Il faut donc de temps à autre, enlever la hausse pour réaliser cette opération. Si le praticien a soin de
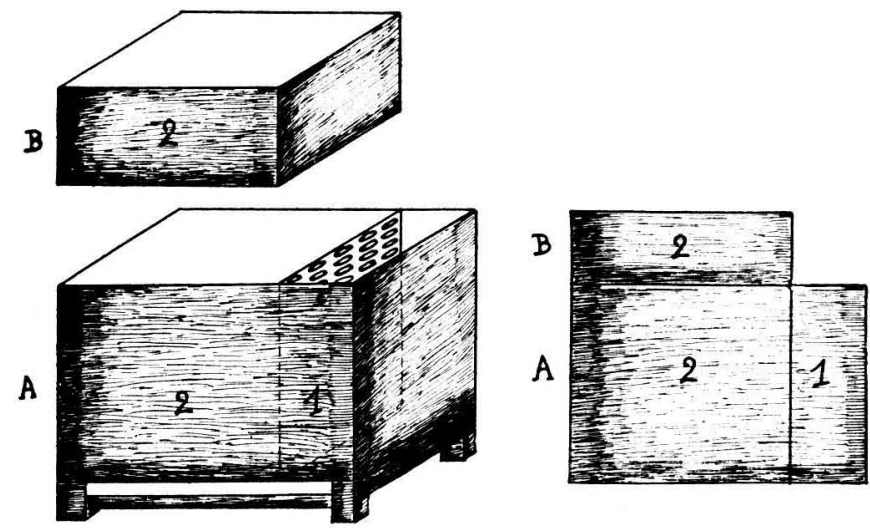

Fig. 2. - Ruche Dadant i 2 cadres $(A)$ et sa hausse $(B)$. La hausse ne couvre que la partie non orpheline de la ruche.

placer des cadres contenant non seulement des larves mais aussi des oufs, il est assuré d'avoir un compartiment orphelin garni de larves durant 9 jours au moins. Il suffit donc d'enlever la hausse une fois tous les 9 jours. C'est là une complication banale, d'importance tout à fait secondaire. Ires cadres porte-cellules peuvent ainsi être introduits dans les ruches et retirés sans perturber l'activité de la plupart des Abeilles.

\section{Résultats.}

Fin I958, 1'année n'a donné lieu qu'à de faibles récoltes ; nous avons pourtant cessé tout nourrissement sur 30 ruches Dadant Io et I 2 cadres du I er mai au I er août. Nous ayons placé les hausses dès qu'elles ont semblé nécessaires et à notre grande surprise, alors qu'un nourrissement avait semblé auparavant indispensable pour faire de la gelée royale, nous avons récolté en moyenne $5 \mathrm{~kg}$ de miel par ruche. 
I,es ruches horizontales sont évidemment plus pratiques pour cette production simultanée, mais peu d'apiculteurs possédaient de telles ruches avant l'exploitation de la gelée royale et notre technique permet d'utiliser un matériel qui à priori, peut sembler inadapté.

\section{Le rendement en gelée roviale.}

Une question très inportante se pose alors : nous avons récolté du miel en voulant produire de la gelée royale, mais quelle a été la production de gelée royale? N'a-t-elle pas été moindre en supprimant le nourrissement? A cette question, nouts ne pouvons pas répondre. Nous ne disposions pas, en effet, de ruches témoins, nourries en mai, juin, juillet. Toutefois nous savons que ce qui importe surtout pour la production de gelée royale, c'est la présence de jeunes Abeilles dans une ruche. Or, il suffit de noter que la ponte a été normale pendant toute la période de production pour admettre qu'elles devaient se trouver en nombre suffisant.

De plus, les quantités de gelée royale moyennes produites sont très satisfaisantes en ce qui nous concerne. Greffant 60 à 70 larves par ruche, nous avons récolté pendant les mois de juin, juillet, toujours plus de ro $g$ de moyenne par ruche. Par opposition aux praticiens qui, pour produire de la gelée royale, continuent à faire chaque jour d'ennuyeuses manipulations, nous n'effectuons plus de recherche de reine, nous n'avons plus"d'Abeilles à secouer et à restituer à la souche et très peu à nourrir. Les manipulations que nous espérons réduire encore sont simplifiées à un point tel que la production de gelée rovale devient un travail de laboratoire. Nous passons en effet beaucoup plus de temps au "greffage " des larves, préparation des cellules et récolte de la gelée royale, que dans les ruchers.

\section{La production de paquets d'abeilles.}

I.es ruches latérales offrent un autre avantage qu'il convient de signaler ici : les colonies occupent facilement au cours d'une saison apicole les $\mathrm{I} 7$ cadres que nous avons mis à leur disposition. C'est dans ces ruches que nous avons puisé durant toute la saison les larves servant au "picking". Ces colonies énormes peuvent sans inconvénient à la fin de la saison, fournir 4 et 5 cadres avec les abeilles qui les couvrent; réunis 2 par 2, ces fragments de colonies auxquelles il suffit de donner une reine, constituent des colonies normales qui, l'année suivante sont utilisées sans distinction de provenance. Cette pratique, possible aussi avec les ruches Dadant Io et I2 cadres est toutefois plus dangereuse.

Au moment où nous rédigeons cette note, nous essayons d'ajouter à ces récoltes, la récolte du pollen. Nous pouvons déjà affirmer que la 
chose est possible, nous préciserons nos résultats par ailleurs. Au cours de la même année I958, certains apict1teurs producteurs de Gelée royale ont d'ailleurs récolté simultanément du pollen et de la gelée royale, avec des résultats qu'ils considèrent comme satisfaisants.

\section{REFERENCES BIBLIOGRAPHIQUES}

Vuiliaume (M.). - Production de gelée royale. Le starter perpétuel. L'Apiculteur (section scientif.), nov. I955.

Vuiliaume (M.). - Élevage de reines. Production de gelée rovale. L'Apiculteur (section scientif.), avr. I957.

Vuinitaume (M.). - Techniques d'élevage des reines. Am. Abeille, 3, I958.

VuiridAume (M.). - Contribution à la psychophysiologie de l'élevage des reines chez les abeilles. I. Insectes sociaux, VI, 2, I957. - II. Ann. Abeille, 2, I959. 